\title{
La léthargie chez les Chiroptères cavernicoles d'Afrique Centrale
}

\author{
par \\ Michel ANCIAUX de FAVEAUX*
}

\author{
SUMMARY \\ Lethargy in the cavernicolous Chiroptera in Central Africa
}

From his personal research undertaken in the subterranean field (natural and artificial cavities) in Shaba (ex-Katanga, in S.E. Zaire) and Rwanda, the author briefly defines the macroclimate of the prospected regions as well as the microclimate of the subterranean habitat (humidity and temperature).

A reversible hypothermia has been noticed in the dry season only (from May till August) in eleven species of troglophile Chiroptera belonging to the following families: Rhinolophidae (7 species of Rhinolophus), Hipposideridae (only Hipposideros ruber) and Vespertilionidae (Miniopterus inflatus rufus, Miniopterus schreibersi arenarius \& M.s. natalensis, Myotis tricolor).

No sign of lethargy has been noticed in the Megachiroptera (Lissonycteris angolensis, Rousettus aegyptiacus leachi), Emballonuridae (Taphozous perforatus sudani), Hipposideridae (Cloeotis percivali australis) or Nycteridae (3 species of Nycteris).

There could be correlations between lethargy and breeding if one takes into account the phenomena of late ovulation and delayed implantation.

The entry into lethargy is not caused by the scarcity of food. It does not concern all the individuals of a colony or in various populations of a cave. The degree of humidity appears to be more important than the temperature as far as the conditions for hibernation are concerned.

D'octobre 1954 à fin juin 1965, nos investigations personnelles se sont localisées dans le S.E. Zaïre (Shaba = ex-Katanga); nous avons axé nos observations sur la biologie, l'écologie et la parasitologie des Chiroptères, tout en collectant des invertébrés cavernicoles terrestres et aquatiques (Anciaux, 1956, 1958, 1960 a \& b, 1964, 1965).

De novembre 1965 à juin 1968, nous avons étudié les Chiroptères du Rwanda.

Une synthèse de nos recherches a fait l'objet de deux thèses de Doctorat (Anciaux, 1972 a \& b).

* Institut des Sciences Biologiques Département Vétérinaire Laboratoire de Parasitologie Université de CONSTANTINE (Algérie). 


\section{CAVITÉS EXPLORÉES}

\section{A. Dans le Haut-Katanga (Shaba)}

Nous avons visité, souvent à plusieurs reprises, 52 grottes calcaires et galeries souterraines artificielles qui se répartissent comme suit:

- région de Lubumbashi (ex-Elisabethville): grotte de Baya et 5 caniveaux d'évacuation d'eaux sous les routes;

- région de Likasi (ex-Jadotville): grotte de Kakontwe, grottes de Pempéré (au nombre de 14; surtout grotte Mwanga) et une galerie minière (cuivre);

- région de Kambove-Shinkolobwe: Mulungwishi (4 grottes), Tantara (6 grottes), Kalumbu (Kabolela), Kambove (2 grottes dans le domaine Swanepoel) et une galerie minière (Shinkolobwe);

- région de Lubudi: grottes de Kyantapo, Kyamakonde, Kandu, Mpopola, Mulonga, Kyasala, Lusolo et Kaboyaboya;

- loin de centres urbains: Kakanda (grotte de Kondo et galerie minière), Mwela-Pande (grotte Tshamakele), Mont Kibwe, Kiwakishi, Parc National de l'Upemba (grotte Kasoma), Tshamalenge, Mwadingusha (galerie le long d'une conduite forcée).

Nous avons publié les détails sur la localisation et sur la description de ces grottes (Anciaux, 1964, 1965).

Certaines grottes verticales (gouffres) sont d'accès très difficile: leur exploration a été rendue possible grâce à la collaboration de nos amis de la Société de Spéléologie du Katanga qui ont mis leur enthousiasme à notre service, spécialement de 1958 à 1960.

\section{B. Au Rwanda}

Le milieu souterrain se caractérise par trois types de cavités:

\section{Petites grottes ou fissures granitiques}

- Grotte de Birambo (coordonnées $02^{\circ} 28^{\prime} \mathrm{S}$. \& $29^{\circ} 40^{\prime} \mathrm{E}$.): faille rocheuse sur la rive gauche de la rivière Mwogo, commune de Kinyamakara, près de Butaré (= ex-Astrida).

- Grotte de Nyumba (coordonnées $02^{\circ} 38 \mathrm{~S}$. \& $29^{\circ} 42^{\prime} \mathrm{E}$.): petite cavité à mihauteur d'une profonde dépression; couloir d'environ un mètre de haut, d'une dizaine de mètres de long, terminé par une petite salle très exiguë et obscure avec une flaque d'eau permanente.

- Fissures dans de gros blocs granitiques érodés à Nyankokoma (colline Nyabinyenga: coordonnées $02^{\circ} 18^{\prime} \mathrm{S}$. \& $29^{\circ} 41^{\prime} \mathrm{E}$.), au N.W. de Nyanza; nous y avons capturé un Molossidé assez rare, Tadarida fulminans (Thomas, 1903): une femelle dans une colonie d'une dizaine d'individus, le 6 février 1966. Sous les rochers existent également des "trous à hyènes".

2. Grottes volcaniques (régions de Kisenyi et de Ruhengeri)

Trois grottes de lave à Musanse (près de Ruhengeri: coordonnées $01^{\circ} 30^{\prime} \mathrm{S}$. 
$\& 29^{\circ} 38^{\prime}$ E.): tunnels plus ou moins rectilignes, disposés suivant un axe nordsud, à quelques mètres sous la surface du sol, avec une hauteur moyenne de 3 à $5 \mathrm{~m}$; leur parcours est fréquemment interrompu par des éboulements qui ont déterminé de petites dépressions envahies par des épineux; sur les bords de ces pseudo-dolines, les Dracaena constituent un bon repère pour localiser les entrées des grottes.

Leur formation relativement récente (tout au plus un million d'années) a empêché l'installation d'une vraie faune cavernicole (Jeannel, 1961).

\section{Cavités artificielles}

- Anciennes galeries de prospection minière dans des gisements de cassitérite, à Lugarama (coordonnées $01^{\circ} 44^{\prime} \mathrm{S}$. \& 30 27'E.; alt. $1.500 \mathrm{~m}$ ).

- Caniveaux sous route pour l'évacuation des eaux de pluie, à Kakitumba (coordonnées $01^{\circ} 03^{\prime} \mathrm{S}$. \& $30^{\circ} 28^{\prime} \mathrm{E}$.), à la frontière de l'Uganda.

\section{CLIMATOLOGIE}

\section{A. Régime climatique général (macroclimat)}

\section{Katanga}

Le climat haut-katangien, assez homogène, appartient au type Soudanoguinéen (tropical semi-humide) au point de vue de la répartition des pluies. L'indice pluviométrique moyen se situe entre 1.000 et $1.300 \mathrm{~mm}$. A Lubumbashi (alt. $1.229 \mathrm{~m}$; coordonnées $11^{\circ} 39^{\prime} \mathrm{S}$. \& $27^{\circ} 28^{\prime} \mathrm{E}$.), la température moyenne annuelle est de 20,5 à $21,4^{\circ} \mathrm{C}$; pour la température moyenne mensuelle, les minima sont compris entre 15,2 et 16,6 tandis que les maxima varient entre 24,3 et $25^{\circ} \mathrm{C}$.

Le rythme des saisons peut se résumer comme suit: d'octobre à avril (mai), saison des pluies (parfois interrompue par une petite saison sèche, en janvier); de mai à septembre (octobre), grande saison sèche, aride, particulièrement fraîche en mai-juin (en raison de l'altitude moyenne supérieure à $1.000 \mathrm{~m}$ ).

Cette aire climatique se prolonge vers le Nord-Est (Tanzanie occidentale), vers l'Ouest (Angola) et vers le Sud (Zambie).

\section{Rwanda}

Le sous-climat ruandien relève du type Ougandien; il est caractérisé par 7 mois très pluvieux, avec une saison sèche de juin à septembre. La température moyenne annuelle se situe entre 15 et $18^{\circ} \mathrm{C}$ (en raison de l'altitude moyenne d'environ $1.400 \mathrm{~m}$ ).

Régime des saisons: petite saison sèche en janvier-février; grande saison sèche de la mi-juin à septembre; saison des pluies de la fin septembre à la mi-juin. 


\section{B. Microclimat dans le domaine souterrain}

1. Humidité relative

a) Katanga

De 95 à 100\%, sauf dans les grottes sèches (Baya, Kasowena et Kiwakishi).

b) Rwanda

- Grottes granitiques (Birambo, Nyumba): plus de 95\% dans les salles terminales.

- Grottes de lave (Musanse): dans la grotte I, de 84 à $90 \%$, avec beaucoup d'eau suintant de la voûte; dans la grotte II, $86 \%$.

\section{Température (en degrés centigrades)}

En règle générale, la température d'une grotte correspond à la température extérieure moyenne annuelle.

Alors que dans les grottes peuplées de troglobies en Europe et en Afrique du Nord, la température moyenne est de $10,7^{\circ}$ (Jeannel, 1926), celle des grottes du Zaïre ( $=$ ex-Congo belge) est beaucoup plus élevée (variant entre 18 et $27^{\circ}$ ), ce qui explique la pauvreté de la faune troglobie terrestre (Leleup, 1956).

\section{a) Katanga}

\section{$1^{\circ}$ Grottes de grandes dimensions}

Dans la grotte de Kakontwe (avec lac souterrain): le 8 mai 1965, 21,5 (air) et $22^{\circ}$ (eau); le 15 mai 1957, 23 (air) et $23,5^{\circ}$ (eau); le 12 juillet 1957, $24^{\circ}$ air (à 9 h. du matin, température extérieure $9,5^{\circ}$ ); le 11 septembre 1957, 22 (air) et $23,4^{\circ}$ (eau).

Nous avons enregistré les températures de l'air dans 11 grottes; ces mesures varient entre 19,5 et $24^{\circ}$ au cours de l'année, et se répartissent comme suit: février $\left(24^{\circ}\right)$, avril $\left(22^{\circ}\right)$, mai $\left(21,21,5,22\right.$ et $\left.23^{\circ}\right)$, juin $\left(21,5^{\circ}\right)$, juillet $\left(19,5,20,22,23\right.$ et $\left.24^{\circ}\right)$, septembre $\left(22^{\circ}\right)$.

L'isolement microclimatique de quelques grottes n'est pas totalement réalisé: nous avons noté des températures plus basses en saison sèche (de mai à juillet) où les nuits sont très fraîches à l'extérieur (jusqu'à 3 et $2^{\circ} \mathrm{C}$.).

$2^{\circ}$ Grottes moins profondes (massif de Pempéré)

- Grotte Mwanga: le 30 juin 1964, $13^{\circ}$ en bas de la cheminée d'accès, et $15^{\circ}$ dans la salle terminale.

- Grotte Katembavikulu: le 30 juin $1964,16^{\circ}$ (air) et $18,5^{\circ}$ (eau); le 31 août 1958, 23 (air) et $19^{\circ}$ (eau). Le fossé d'eau (nappe phréatique) avait, le 3 octobre 1959, un pH de 7,5 en eau claire, et de 7,2 en eau boueuse; il est peuplé de très nombreux Amphipodes troglobies (Ingolfiella leleupi Ruffo, 1951).

b) Rwanda

$1^{\circ}$ Grotte de Nyumba: le 1 février $1966,23^{\circ}$ (air), à 5 mètres de l'entrée. 
$2^{\circ}$ Grottes volcaniques de Musanse

- Grotte I (7 juin 1966): vers le milieu du tunnel, $84 \%$ d'humidité; thermomètre $\left(\sec 18,3^{\circ}\right.$, humide $\left.16,6^{\circ}\right)$. Aux deux tiers de la galerie, $90 \%$ d'humidité; thermomètre ( $\sec 17,7^{\circ}$, humide $\left.16,6^{\circ}\right)$.

- Grotte II ( 8 juin 1966): humidité de $86 \%$; thermomètre ( $\sec 17,7^{\circ}$, humide $16,1^{\circ}$ ).

Isolement thermique fort relatif.

$3^{\circ}$ Cavités artificielles (Verschuren, 1965)

- Caniveau sous route (Kakitumba): le 9 février 1960, à 11 h., température extérieure (max. 28,5 et $\min .20^{\circ}$ ); dans le drain, $\max .-\min .27$ et $20^{\circ}$.

- Mine de cassitérite à Lugarama: le 30 avril $1960,23^{\circ} \mathrm{C}$.

\section{Remarque}

Les individus léthargiques sont habituellement localisés dans des renfoncements où l'air est presque immobile.

\section{CHIROPTẼRES TROGLOPHILES DU KATANGA ET DU RWANDA}

En dehors de la saison sèche nous n'avons trouvé aucune espèce en vraie léthargie.

En saison froide (sèche), seuls les Minioptères manifestent une léthargie profonde qui affecte la presque totalité des individus composant les colonies. Chez les autres espèces, on observe rarement des colonies entières en état de torpeur; le sommeil hypothermique atteint quelques individus qui s'isolent alors des colonies; d'autres, même solitaires, restent actifs.

Nous n'avons décelé aucun signe de léthargie chez les 7 espèces cavernicoles suivantes: Lissonycteris angolensis (Bocage, 1898), Rousettus aegyptiacus leachi (A. Smith, 1829), Taphozous perforatus sudani (Thomas, 1915), Nycteris aethiopiea Dobson, 1878, Nycteris macrotis Dobson, 1876, Nycteris thebaica E. Geoffroy, 1818 et Cloeotis percivali australis Roberts, 1917.

Nos observations positives concernent les familles suivantes: Rhinolophidae, Hipposideridae et Vespertilionidae.

\section{A. RHINOLOPHIDAE}

1. Rhinolophus blasii empusa (Andersen, 1904)

Un mâle, profondément endormi, au milieu d'une colonie léthargique d'Hipposideros ruber (des deux sexes), le 31 mai 1962, dans la grotte de Kondo (coordonnées $10^{\circ} 47^{\prime} \mathrm{S}$. \& $26^{\circ} 36^{\prime} \mathrm{E}$.).

2. Rhinolophus clivosus zuluensis Andersen, 1904

Une femelle, à l'écart d'autres Rhinolophidés (un Rhinolophus hildebrandti mâle et deux Rhinolophus landeri lobatus femelles) également endormis, le 
21 juin 1959, dans la grotte Mwanga, à Pempéré (coordonnées $10^{\circ} 58^{\prime} \mathrm{S}$. \& $26^{\circ} 39^{\prime}$ E. $)$.

\section{Rhinolophus fumigatus exsul Anderson, 1905}

Au Katanga, colonies éveillées: le 22 avril 1959, 4 mâles et 7 femelles (parmi une cinquantaine d'individus) dans la grotte de Kondo; au sein d'une trentaine d'animaux de la même espèce, nous avons capturé 1 mâle et 6 femelles, le 18 décembre 1957, le long d'une conduite forcée, à Mwadingusha (coordonnées $10^{\circ} 45^{\prime} \mathrm{S}$. \& $27^{\circ} 14^{\prime} \mathrm{E}$.).

Au Rwanda, dans les grottes de lave de Musanse: grotte I, 4 mâles et 3 femelles, le 7 juin 1966; grotte III, 1 mâle et 2 femelles, le 10 juin 1966; aucun Chiroptère dans la grotte II, le 8 juin 1966. Tous ces Rhinolophes étaient bien endormis et isolés les uns des autres, très loin de la colonie de Rousettus aegyptiacus leachi, mais relativement près de Miniopterus schreibersi arenarius en léthargie.

\section{Rhinolophus hildebrandti Peters, 1878}

Beaucoup d'individus en sommeil hypothermique, parmi quelques autres éveillés, dans les grottes du Katanga: à Kakontwe, surtout des mâles, les 8 et 12 juillet 1957; à Pempéré, seulement des mâles, les 19 juin 1957, 21 juin 1959 et 7 août 1960; à Kalumbu (alt. 1.595 m.; coordonnées $10^{\circ} 50^{\prime}$ S. \& $26^{\circ} 39^{\prime}$ E.), un mâle, le 1 juillet 1959 ; dans la grotte Kyasala (alt. $1.300 \mathrm{~m}$.), à $6 \mathrm{~km}$ de Lubudi (coordonnées $09^{\circ} 56^{\prime} \mathrm{S}$. \& $25^{\circ} 58^{\prime} \mathrm{E}$.), une colonie composée des deux sexes, le 28 juillet 1957. Nous n'avons vu et capturé que des femelles très actives, le 4 juin 1960, dans la grotte au Ruisseau, à Tantara (coordonnées $11^{\circ} 02^{\prime} \mathrm{S}$. \& $26^{\circ} 29^{\prime} \mathrm{E}$.). Dans la grotte de Kakontwe (coordonnées $10^{\circ} 59^{\prime}$ S. \& $26^{\circ} 42^{\prime}$ E.; alt. 1.294 m.), la plupart des Rhinolophes étaient fort éveillés et isolés les uns des autres (sauf un groupe de 3), le 28 juin 1960.

5. Rhinolophus landeri lobatus Peters, 1852

Dans la grotte Kalumbu, un couple en plein sommeil, le 1 juillet 1959, à l'écart de la colonie qui était éveillée.

\section{Rhinolophus simulator Andersen, 1904}

Dans les grottes du Katanga: à Kakontwe (11 juin 1960) et à Lubudi (18 juillet 1962), des individus (des deux sexes) isolés dormaient dans des fissures de la voûte ou dans les plis des draperies; dans la grotte Katembavikulu (Pempéré), ils étaient accrochés au plafond, le 7 août 1960.

\section{Rhinolophus swinnyi Gough, 1908}

Dans les grottes du Katanga: à Pempéré (seulement des femelles, le 21 juin 1959) et à Lubudi (individus des deux sexes, les 18 juillet 1962 et 30 juillet 1957); ces Rhinolophes étaient isolés des colonies, engourdis. 


\section{B. HIPPOSIDERIDAE}

1. Cloeotis percivali australis Roberts, 1917

Parmi les 5 mâles et les 4 femelles provenant des grottes du Katanga: à Tantara, (les 4 juin 1960 et 15 juin 1958), à Lubudi (grotte Kaboyaboya, le 23 juillet 1962) et au Parc National Upemba (grotte Kasoma: coordonnées $09^{\circ} 25^{\prime} \mathrm{S}$. \& $26^{\circ} 37^{\prime} \mathrm{E}$.; le 13 août 1962 ), les solitaires ou les petits groupes étaient constamment en éveil au milieu d'autres espèces de Chiroptères en léthargie.

\section{Hipposideros caffer (Sundevall, 1846)}

Espèce signalée au Rwanda (Verschuren, 1965).

Au Katanga, aucune observation, car nous attribuons notre matériel à l'espèce Hipposideros ruber (Noack, 1893).

\section{Hipposideros commersoni (E. Geoffroy, 1813)}

Au Katanga, 5 spécimens d'Hipposideros commersoni gigas (Wagner, 1845) ont été récoltés dans 4 localités par d'autres naturalistes; deux individus, accrochés à moins d'un mètre de la surface du lac souterrain de Kakontwe, le 5 juillet 1948 (Leleup, 1956). Aucune donnée sur la léthargie.

Au Rwanda, un seul Hipposideros commersoni marungensis (Noack, 1887) à Kisenyi (Schouteden, 1948).

\section{Hipposideros ruber ruber (Noack, 1893)}

Nos récoltes au Katanga nous ont fourni 87 mâles et 53 femelles, durant les mois de janvier à août.

Les seules observations positives que nous avons faites sur la torpeur hypothermique des Hipposidéridés concernent Hipposideros ruber:

- des individus isolés, profondément endormis, dans des fissures du niveau supérieur de la grotte de Kakontwe, les 18 mai 1958 et 28 juin 1960;

- une colonie en léthargie (capture de 31 mâles et de 10 femelles), le 31 mai 1962, dans la grotte de Kondo.

\section{VESPERTILIONIDAE}

1. Miniopterus inflatus rufus Sanborn, 1936

Rares individus ( 2 mâles et 1 femelle), somnolents, dans des fissures de la grotte Kasoma (P. N. Upemba), le 13 août 1962, en compagnie de Miniopterus schreibersi natalensis.

\section{Miniopterus schreibersi arenarius Heller, 1912}

Détermination de V. Aellen ("in littera", 2 février 1971). Au Rwanda, deux mâles en état de torpeur complète, le 7 juin 1966, accrochés à la voûte suintante de la grotte de lave I (Musanse), et assez éloignés de Rhinolophus fumigatus. 


\section{Miniopterus schreibersi natalensis (A. Smith, 1834)}

Nos observations sont basées sur une très importante collection ( 245 mâles et 267 femelles) et sur un gros effectif (341 mâles et 260 femelles) de Minioptères qui ont été bagués par nous, et libérés assez loin de leurs gîtes (expériences de dépaysement au Katanga).

La presque totalité de nos captures (effectuées au sein de grandes colonies) a été faite durant les mois d'avril à août, $c$-à-d durant la saison sèche, dans les quartiers d'automne et d'hiver (20 grottes et 2 galeries artificielles).

Colonies léthargiques dans les grottes du Katanga

Grotte de Kakontwe

- 8 mai 1965: deux ou 3 Minioptères par fissure, nombreux individus sur les parois (un seul groupe de 4).

- 18 mai 1958: premiers individus bien endormis.

- 20 mai 1962: mâles isolés ou en petits groupes.

- 28 juin 1960: seuls les Minioptères (des deux sexes) dormaient; tous les autres Chiroptères remuaient ou volaient.

- 8 et 12 juillet 1957: groupes de $2,4,5,20$ et jusqu'à 50 individus étroitement serrés sur les parois ou dans les fissures (plusieurs essaims formés uniquement de mâles; d'autres comprenant les deux sexes).

L'entrée en léthargie a lieu durant la première quinzaine de mai.

Grottes de Pempéré

- 21 juin 1959: importante colonie formant plusieurs gros essaims (des deux sexes) fixés contre les parois; réveil très lent lors de la capture (grotte Mwanga).

- 28 juin 1964: un très grand nombre (surtout des femelles) en léthargie (grotte Kasowena).

- 30 juin 1964: essaim composé des deux sexes (grotte Mwanga).

- 7 août 1960: quelques individus en état de torpeur; le gros contingent de la colonie avait déserté la grotte Mwanga.

Les Minioptères sont seulement présents de juin à août dans la grotte Mwanga (quartier d'hiver).

Galerie minière de Likasi

- 3 juin 1958: colonie endormie (des deux sexes).

- 26 août 1958: gros essaims de Minioptères légèrement endormis (capture de 24 mâles et de 72 femelles).

Grottes de Lubudi

- 18 juillet 1962: colonie en léthargie (seulement des mâles) dans la grotte Kyamakonde.

- 25 et 28 juillet 1957: colonies formées des deux sexes, dans les grottes Kandu, Kyasala et Lusolo.

Grotte de Baya (coordonnées $11^{\circ} 52^{\prime} \mathrm{S}$. \& $27^{\circ} 07^{\prime} \mathrm{E}$.; alt. $1.300 \mathrm{~m}$.)

- 14 mai 1956: colonie (des deux sexes) bien endormie.

Grotte Kasoma (Parc National de l'Upemba)

- 13 août 1962: individus des deux sexes, à peu près inertes, en compagnie de Miniopterus inflatus rufus, dans des fissures pariétales; un "harem" composé d'un mâle et de deux femelles dans une fissure. 
Les Minioptères étaient rares de septembre à mars: seulement dans 4 grottes. En août-septembre (fin de la saison sèche), une migration aurait lieu vers les gîtes de reproduction (qui nous restent inconnus). Les mises bas non simultanées ont lieu au début de la saison des pluies, avec un décalage suivant des latitudes de plus en plus méridionales: mi-octobre (Katanga), début de novembre (Zambie \& S.W.Afrique), de la fin octobre à la fin novembre (Transvaal) et au début de décembre (Natal).

Une fois la lactation terminée, les colonies de reproduction se disloquent vers la fin janvier, en vue de regagner les quartiers d'hiver entre fin février et début avril.

La copulation aurait lieu, en avril, avant la période de léthargie; durant le sommeil hypothermique, il y aurait une implantation différée d'environ 3 mois, comme c'est le cas en Europe pour Miniopterus s. schreibersi (Peyre \& Herlant, 1963; Saint Girons \& al., 1969) et en Australie pour Miniopterus schreibersi blepotis (Dwyer, 1963). La gestation ne débuterait que vers la fin juillet (fin de l'hibernation en Afrique australe).

\section{Myotis (Myotis) tricolor (Temminck, 1832)}

Solitaires au fond d'étroites fissures, en état de torpeur profonde, dans la grotte au Ruisseau, Tantara (un mâle, le 4 juin 1960) et dans deux grottes de Lubudi ( 2 femelles, le 18 juillet 1962; un mâle, le 30 juillet 1957). Un mâle, très peu actif, le 22 septembre 1957, dans la grotte de Kakontwe.

Nous avons observé deux cas d'étroit commensalisme avec Miniopterus schreibersi natalensis, dans la région de Lubudi: un Minioptère mâle au fond d'une fissure derrière 2 Myotis tricolor femelles, grotte de Kyamakonde, le 18 juillet 1962; un Myotis tricolor mâle avec plusieurs Minioptères, grotte de la Mulonga, le 30 juillet 1957.

\section{DISCUSSION}

Au Parc National Albert (Kivu: Zaïre), dans la grotte volcanique de Djomba (lat. $01^{\circ} 20^{\prime} \mathrm{S}$.), une colonie de Rhinolophus clivosus en semi-léthargie, le 27 juin 1960: température de 15 et $15,5^{\circ} \mathrm{C}$, humidité proche de $100 \%$; température anale: $20,5^{\circ}$ chez un Rhinolophe endormi, 38, $2^{\circ}$ chez un individu actif (Verschuren, 1967). Dans cette même grotte, un Myotis tricolor en semi-léthargie, le 24 octobre 1960 (c-à-d en saison chaude), suspendu librement au milieu d'une colonie éveillée de Rhinolophus clivosus femelles avec de petits jeunes (Verschuren, 1967).

Aucun signe de léthargie diurne chez Hipposideros caffer ni chez Hipposideros commersoni gigas, au Gabon; la thermorégulation varie seulement de quelques degrés (entre 31,5 et $35,5^{\circ}$ ) suivant les phases de repos et d'activité. Des expériences d'hibernation provoquées ont montré que les grands Hipposideros (H.gigas) restent constamment éveillés (même sous $4^{\circ} \mathrm{C}$ ) en activant leur métabolisme, tandis que les petits Hipposideros (H.caffer) tombent dans un état d'hypothermie irréversible et meurent après 8 heures 
(Brosset, 1969). Il n'en est pas de même pour Miniopterus inflatus: cinq individus refroidis expérimentalement à $4^{\circ} \mathrm{C}$, pendant 32 heures, ont montré une hypothermie profonde réversible; remis dans une température ambiante normale, ils ont repris leur vol (Brosset, 1969).

Chez Miniopterus schreibersi arenarius, Verschuren (1967) a étudié le comportement léthargique dans deux grottes de lave du Parc National Albert: au Mont Muvo (lat. 01 $1^{\circ} 14^{\prime} \mathrm{S}$.; alt. $1.950 \mathrm{~m}$.), 4 mâles en profonde léthargie, le 20 septembre 1959, réveillés 20 minutes après leur capture (minima nocturnes de $13^{\circ}$ dans la grotte et de $11^{\circ}$ à l'extérieur); à Mugongo (lat. $01^{\circ} 30^{\prime} \mathrm{S}$; ; alt. $2.280 \mathrm{~m}$.), un mâle et 2 femelles en léthargie presque totale, le 28 juin 1960, parmi des Rhinolophes très éveillés (température de la grotte, $15^{\circ}$; température anale chez un Minioptère endormi, $25^{\circ}$, et $37,2^{\circ}$ chez un autre réveillé).

Chez Miniopterus schreibersi natalensis, au Transvaal (Van Der Merwe, 1970, 1973 a, b \& c), dans la région d'Uitkomst, deux grottes sont fréquentées par les Minioptères en automne et en hiver:

American Cave: lieu probable de l'accouplement, déserté ensuite lors de l'hibernation; de janvier à avril, la température est stable (autour de $17^{\circ} \mathrm{C}$ ) et l'humidité varie entre 89 et $95 \%$;

Long One Cave: grotte d'hibernation (de mai à juillet). Les températures, plus basses que dans American Cave, seraient plus favorables à l'hibernation; par contre, il y a de très grandes variations dans le taux d'humidité, qui est généralement plus bas (entre 33 et $75 \%$ ). Une température basse (même variable) serait donc un élément plus important qu'une haute humidité, dans le choix du site d'hibernation (Van Der Merwe, 1970). Il n'y a pas de période continue de léthargie: l'activité des Minioptères consiste surtout en déplacements à l'intérieur de la cavité, mais elle comporte également des fluctuations du nombre d'individus causées par des sorties vers d'autres grottes plus froides (à la suite de changements de température dans Long One Cave) et par des retours plus ou moins massifs (Van Der Merwe, 1970 \& 1973). Nous avons fait les mêmes observations, avec d'autres espèces de Chiroptères cavernicoles, en Belgique (Anciaux, 1948). La différence entre les températures de Long One Cave et celles des Minioptères est de $0,2^{\circ}$ durant la plus grande partie de l'hibernation, et de $3,1^{\circ} \mathrm{C}$ à la fin de la léthargie hivernale. La température anale des chauves-souris est toujours légèrement plus haute que celle de l'environnement (Van Der Merwe, 1970).

Des corrélations entre la reproduction et la léthargie nous semblent possibles. En effet, les phénomènes d'ovulation retardée et d'implantation différée peuvent être des adaptations au régime des saisons (sèches ou froides). Chez Miniopterus schreibersi, l'implantation différée nous paraît généralisée sous tous les climats; elle a donc une base essentiellement génétique qui conditionne, probablement, une mise en hibernation (métabolisme ralenti). Chez Myotis tricolor, une ovulation retardée (amenant une fécondation différée) semble possible, comme chez Myotis myotis (Anciaux, 1954; Herlant, 1958); la ponte ovulaire aurait lieu au printemps (après la fin de la période léthargique), stimulée par l'hormone de lutéinisation produite à la fin de l'hibernation 
(Herlant, 1956). Chez Hipposideros caffer guineensis et chez Rhinolophus landeri, au N.Nigeria (lat. $10^{\circ}$ N.), Menzies (1973) a démontré une implantation différée de décembre à janvier, c-à-d durant la saison sèche, sans toutefois signaler des cas de léthargie; la gestation serait ainsi retardée, pour que la mise bas ait lieu à une époque où l'humidité augmente (favorisant ainsi une nourriture plus abondante pour les jeunes).

Les genres Rhinolophus et Miniopterus sont cosmopolites et sont capables de peupler les régions froides, grâce à leur faculté d'entrer en léthargie (Brosset, 1969). Nous pensons pouvoir y inclure les genres Hipposideros et Myotis. Toutefois, Brosset (1969) soutient que le genre Hipposideros, qui est localisé dans les régions chaudes de l'Ancien Monde, ne peut pas hiberner, car il n'y a pas d'hypothermie réversible; au Gabon, Hipposideros commersoni gigas n'hiberne jamais spontanément, et sa température interne est soumise à un rythme circadien: de $31-32^{\circ}$ le matin à $36^{\circ} \mathrm{C}$ vers 18 heures, c-à-d quelque temps avant le depart pour la chasse nocturne (Saint Girons \& al., 1969). Nos observations sur la léthargie (avec réchauffement lors du réveil) chez Hipposideros ruber au Katanga, obligent à revoir le statut thermorégulateur des Hipposidéridés. Myotis tricolor, d'après nos observations et d'après celles de Verschuren (1967), peut hiberner spontanément.

Le processus de torpeur hypothermique (léthargie plus ou moins profonde) chez les Chiroptères tropicaux, n'atteint pas toute une population chez une espèce donnée; en effet, on peut voir des individus éveillés et d'autres endormis dans une même grotte, et dans des conditions hygrothermiques semblables; il y aurait donc des "rythmes individuels dépendant de stimulations internes dont la nature est encore inconnue" (Brosset, 1961).

Chez les espèces insectivores, ce serait la privation de nourriture qui déclencherait le processus léthargique (Heim de Balsac, 1936; Dwyer, 1971). Ce n'est pas notre opinion: en effet, au Katanga, où il ne pleut presque jamais durant la saison sèche et où les nuits sont très fraîches, nous avons observé plusieurs espèces (susceptibles d'endurer un état léthargique) qui chassaient régulièrement quelques rares insectes près des grottes ou sur de plus grandes distances.

La fonction des essaims durant la léthargie (spécialement chez les Minioptères) est de stabiliser la température à un degré optimal, en vue de réduire les effets de changements thermiques; elle peut aussi limiter la perte d'eau évaporée et aider à maintenir une haute humidité (Davis, 1970).

\section{CONCLUSIONS}

La température des grottes d'Afrique centrale ne provoque pas l'entrée en léthargie; par contre, une humidité assez élevée paraît nécessaire.

L'absence de nourriture n'explique pas le sommeil hypothermique.

$\mathrm{La}$ gestation peut être retardée chez les espèces manifestant une potentialité léthargique.

Dans les colonies de Mégachiroptères cavernicoles (Lissonycteris angolensis 
et Rousettus aegyptiacus leachi), on n'observe jamais de torpeur; cela provient $\mathrm{du}$ fait que ces chauves-souris sont des homéothermes presque parfaits, capables de maintenir une haute température corporelle sous des températures ambiantes variables (même basses), pourvu que l'ingestion régulière de nourriture maintienne leur métabolisme à un haut niveau (Kulzer, 1965).

Les espèces cavernicoles des genres Miniopterus, Myotis et Rhinolophus sont des homéothermes facultatifs, pouvant entrer en léthargie en fonction de conditions écologiques particulières. Peut-être y a-t-il là un fondement génétique?

Hipposideros ruber semble être la seule espèce, chez les Hipposidéridés, qui manifeste une hypothermie réversible.

\section{RÉSUMÉ}

L'auteur expose ses recherches personnelles dans le domaine souterrain (grottes et cavités artificielles), au Katanga (S.E.Zaïre) et au Rwanda. Il définit brièvement le macroclimat des régions prospectées, ainsi que le microclimat des abris souterrains (humidité, température).

Une hypothermie réversible a été observéé seulement en saison sèche (de mai à août) chez 11 espèces de Chiroptères troglophiles appartenant aux familles suivantes: Rhinolophidés ( 7 espèces de Rhinolophus), Hipposidéridés (uniquement Hipposideros ruber) et Vespertilionidés (Miniopterus inflatus rufus, Miniopterus schreibersi arenarius \& M.s. natalensis, Myotis tricolor).

Aucun indice de léthargie n'a été relevé chez les Mégachiroptères (Lissonycteris angolensis, Rousettus aegyptiacus leachi), chez les Emballonuridés (Taphozous perforatus sudani), chez Cloeotis percivali australis (Hipposidéridé) et chez les Nyctéridés (3 espèces de Nycteris).

Des corrélations entre la léthargie et la reproduction semblent possibles, si on tient compte des phénomènes d'ovulation retardée et d'implantation différée.

L'entrée en léthargie n'est pas déterminée par la pénurie de nourriture. Elle ne concerne pas tous les individus au sein d'une même colonie ou dans diverses populations d'une même grotte. Le taux d'humidité apparaît plus important que la température, pour créer les conditions favorables à l'hibernation.

\section{BIBLIOGRAPHIE}

AELLEN, V. - 1971. Communication personnelle. (in littera, 2 février.)

ANCIAUX de FAVEAUX, M. - 1948. Le sommeil hivernal de nos Cheiroptères d'après des observations locales. Bull.Mus. Roy.Hist. Nat.Belgique, 24(25): 1-27.

- 1954. Observations sur une colonie de Murins (Myotis myotis Borkhausen) dans la grotte de Han-sur-Lesse, Belgique. Actes ler Congr. Int. Spéléol., Paris, 3: 245-263; Rassegna Speleol. Ital., 6: 167-183.

- 1956. Aperçu préliminaire sur la spéléologie du Katanga. Bull.Inform.Fédér.Spéléol. Belgique, 3(9): 3-4.

- 1958. Speologica africana. Chiroptères des grottes du Haut-Katanga, Congo belge. Bull. I.F.A.N., 20 A: 263-275.

- 1960a. Reproduction des Chiroptères du Haut-Katanga. Bull.Soc.Spéléol.Katanga, 2: 23-30, 1 pl.

- 1960b. Contribution à l'étude de l'histoplasmose au Katanga. II.- Considérations écologiques sur l'histoplasmose. Ann.Soc.Belge Médec.Trop., 40: 298-303.

- 1964. La faune des grottes du Katanga. Actes III.Congr. Int.Spéléol., Vienne (1961), 3 : 11-21, 2 cartes.

1965. Les grottes du Katanga. Bull.C.E.P.S.I., Elisabethville, no 69: 77-85, 2 cartes. 1972a. Répartition biogéographique et cycles annuels de Chiroptères d'Afrique centrale. 
Thèse Doctorat Université Paris VI, 2 vols., 429 pp., 35 pp. de bibliographie, tableaux, cartes.

- 1972b. Parasitologie des Chiroptères du Continent africain. Thèse annexe Doctorat Université Paris VI, 1 vol. de 276 pp., tableaux, cartes.

- 1973. Essai de synthèse sur la reproduction de Chiroptères d'Afrique (région faunistique Ethiopienne). Period. Biol., 75: 195-199.

BROSSET, A. - 1961. L'hibernation chez les Chiroptères tropicaux. Mammalia, 25: 413-452.

- 1969. Recherches sur la biologie des Chiroptères troglophiles dans le N.E. du Gabon. Biol.Gabonica, 5(2): 93-116.

DAVIS, W. H. - 1970. Hibernation: ecology and physiological ecology. Chapitre VIII: 265-300, in vol.I, Biology of bats, ed. W.A. WIMSATT, Academic Press, New York.

DWYER, P. D. - 1963. The breeding biology of Miniopterus schreibersi blepotis (Temminck) in North-Eastern New South Wales. Austral.J.Zool., 11: 219-240.

- 1971. Temperature regulation and cave-dwelling in bats: an evolutionary perspective. Mammalia, 35: 424-455.

HEIM de BALSAC, H. - 1936. Biogéographie des Mammifères et des Oiseaux de l'Afrique du Nord. Suppl.Bull.Biol.France \& Belgique, Paris, 21: 1-413.

HERLANT, M. - 1956. Corrélations hypophyso-génitales chez la femelle de Myotis myotis. Arch. Biologie, 67(1): 89-180.

- 1958. Corrélations hypophyso-génitales chez la femelle des chauves-souris. Bull.Soc.Zool France, 83: 337-357.

JEANNEL, R. - 1926. Faune cavernicole de la France, avec une étude des conditions d'existence dans le domaine souterrain. Encycl.Entom., 7, Lechevalier, Paris.

- 1961. La Gondwanie et le peuplement de l'Afrique. Ann.Mus.Roy.Afr.Centr., Tervuren, in- $8^{\circ}$, Sc.Zool., 102: 1-161.

KULZER, E. - 1965. Temperaturregulation bei Fledermäusen aus verschiedenen Klimazonen. Z.vergl.Physiol., 50: 1-34.

LELEUP, N. - 1956. La faune cavernicole du Congo belge et considérations sur les Coléoptères reliques d'Afrique intertropicale. Ann.Mus.Roy.Congo Belge, Tervuren, in- $8^{\circ}$, Sc.Zool., 46: $1-171$.

MENZIES, J. I. - 1973. A study of leaf-nosed bats (Hipposideros caffer and Rhinolophus landeri) in a cave in Northern Nigeria. J.Mammalogy, 54(4): 930-945.

PEYRE, A. \& HERLANT, M. - 1963. Ovo-implantation différée et corrélations hypophysogénitales chez la femelle de Miniopterus schreibersii. C.R.Acad.Sci.Paris, 8 juillet, 257: $524-526$.

SAINT GIRONS, H., BROSSET, A. \& SAINT GIRONS, M. C. - 1969. Contribution à la connaissance du cycle annuel de la chauve-souris Rhinolophus ferrum-equinum (Schreber, 1774). Mammalia, 33: 357-470.

SCHOUTEDEN, H. - 1948. Faune du Congo belge et du Ruanda-Urundi. I.-Mammifères. Ann. Mus.Roy.Congo Belge, Tervuren, in- $8^{\circ}$, Sc.Zool., 1 . Chiroptères: pp. 59-94.

VAN DER MERWE, M. - 1970. Social organisation and seasonal activities of the Natal clinging bat, Miniopterus schreibersi natalensis (A. Smith, 1834). M.Sc.Thesis, Univ.Pretoria, South Africa.

- 1973a. Aspects of social behaviour of the Natal clinging bat, Miniopterus schreibersi natalensis. Mammalia, 37(3): 379-389.

- 1973b. Aspects of temperature and humidity in preferred hibernation sites of Mininpterus schreibersi natalensis. Zool.Africana, 8(1): 121-134.

1973c. Aspects of hibernation and winter activities of Miniopterus schreibersi natalensis on the Transvaal Highveld. S.Afr.J.Sci., 69(4): 116-118.

VERSCHUREN, J. - 1965. Exploration du Parc National de la Kagera. Chiroptères. Inst. Parcs Nationaux Congo Belge, Bruxelles, 2ème série, fasc. 1(2): 67-75.

- 1967. Introduction à l'écologie et à la biologie des Cheiroptères. Parc National Albert, Mission F. BOURLIERE \& J. VERSCHUREN, 1957-1961. Ibidem, fasc. 2: 25-65. 